\title{
Some Examples Concerning the Global Markov Property
}

\author{
Robert B. Israel * \\ Department of Mathematics, University of British Columbia, Vancouver, B.C., Canada V6T 1Y4
}

\begin{abstract}
We present examples of interactions of classical lattice systems whose extremal Gibbs states fail to have the global Markov property. One of the examples is translation invariant.
\end{abstract}

\section{Introduction}

Consider a classical lattice system on a lattice $\mathscr{L}$, with a finite-range interaction $\Phi$ (so that the formal Hamiltonian is $\sum_{X \subset \mathscr{L}} \Phi_{X}$ ). Sites $x, y$ will be said to be neighbours if $\Phi_{X} \neq 0$ for some finite $X \subset \mathscr{L}$ containing $x$ and $y$. For any subset $\Lambda \subset \mathscr{L}$, let $\mathscr{F}_{A}$ be the $\sigma$-algebra generated by the spins in $\Lambda$, and let $\partial \Lambda$ be the set of sites not in $\Lambda$ which have at least one neighbour in $\Lambda$. The Gibbs states for the interaction $\Phi$ satisfy the local Markov property:

$E\left(f \mid \mathscr{F}_{\mathscr{L} \backslash \Lambda}\right)=E\left(f \mid \mathscr{F}_{\partial \Lambda}\right)$ for any finite $\Lambda \subset \mathscr{L}$ and bounded $\mathscr{F}_{\Lambda}$-measurable $f$. (1)

The global Markov property is the same statement, but with $\Lambda$ allowed to be infinite.

It was conjectured in [2] that every extremal Gibbs state for $\Phi$ satisfies the global Markov property. A more general conjecture would be that for lattice random fields, the local Markov property plus triviality of the tail field imply the global Markov property. Von Weizsäcker [4] has found a counterexample to the latter conjecture. His random field is not a Gibbs state in the usual sense: it involves constraints rather than interactions. One could construct an interaction whose Gibbs states have similar behaviour, but it would have to be unbounded, growing rapidly enough at infinity so that with high probability all the constraints of von Weizsäcker's process are satisfied. In this note we will construct examples with a bounded, in fact periodic or translation-invariant, interaction whose extremal Gibbs states fail to have the global Markov property.

^ Research supported by Natural Sciences and Engineering Research Council grant A4015 


\section{An Example (Model 1)}

The lattice $\mathscr{L}$ consists of the sites $(i, j, \pm 1 / 2),(i+1 / 2, j, 0)$ and $(i, j+1 / 2,0)$ in $\mathbb{R}^{3}$ for $i, j \in \mathbb{Z}$ (see Figs. 1 and 2). The spin at each site takes the values \pm 1 ; we will denote the spin at $(x, y, z)$ by $s_{x, y}$ if $z=+1 / 2$ (the top layer), $t_{x, y}$ if $z=0$ (middle layer), and $u_{x, y}$ if $z=-1 / 2$ (bottom layer). The interaction is a ferromagnetic three-spin interaction; the Hamiltonian may be written (formally) as

$$
H=J \sum_{i, j \in \mathbb{Z}}\left(s_{i, j} t_{i+1 / 2, j} s_{i+1, j}+s_{i, j} t_{i, j+1 / 2} s_{i, j+1}+u_{i, j} t_{i+1 / 2, j} u_{i+1, j}+u_{i, j} t_{i, j+1 / 2} u_{i, j+1}\right) .
$$

Take for $\Lambda$ the top layer $\mathscr{L} \cap\{z=+1 / 2\}$, so that $\partial \Lambda$ is the middle layer $\mathscr{L} \cap\{z=0\}$.

Theorem. (a) In any Gibbs state of this model for any $J, E\left(s_{i, j} \mid \mathscr{F}_{\partial \Lambda}\right)=0$. (b) For sufficiently large $J$, in an extremal Gibbs state $E\left(s_{i, j} u_{i, j}\right) \neq 0$.

(This implies $E\left(s_{i, j} \mid \mathscr{F}_{\mathscr{L} \backslash A}\right) \neq 0$, so the global Markov property fails.)

Proof. (a) Note that the model has a local "gauge" symmetry: let $\Gamma$ be any rectangle with sides at half-odd-integer values of $x$ and $y$ (e.g. the dashed rectangle in Fig. 1), and flip all the $s$ and $u$ spins inside $\Gamma$ and all the $t$ spins on its boundary. It is easy to see that the Hamiltonian is invariant under this operation. Since it is a local symmetry, all Gibbs states are also invariant. Now if $g(t)$ is any function of the spins in a finite subset $A$ of $\partial \Lambda$, we may take $\Gamma$ so large that $A$ and $(i, j)$ are in its interior. Then the symmetry leaves $g(t)$ unchanged but flips $s_{i, j}$, so $E\left(s_{i, j} g(t)\right)=0$. Since functions of finitely many spins are dense in $L^{2}, E\left(s_{i, j} \mid \mathscr{F}_{\partial A}\right)=0$.

(b) We may explicitly "integrate out" the $t$ spins:

$$
\sum_{t= \pm 1} e^{J t\left(s_{1} s_{2}+u_{1} u_{2}\right)}=\text { const } \cdot e^{K s_{1} s_{2} u_{1} u_{2}} \quad \text { with } \quad K=1 / 2 \log \cosh 2 J .
$$

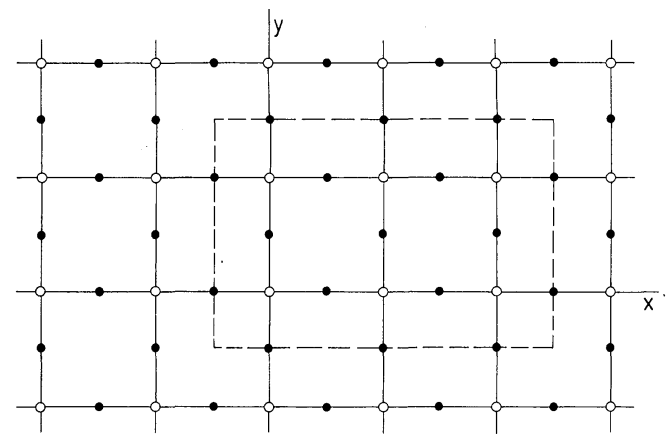

Fig. 1. Top view of $\mathscr{L}$

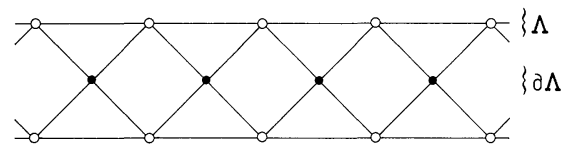

Fig. 2. Side view of $\mathscr{L}$ 
The result is that the variables $\sigma_{i, j}=s_{i, j} u_{i, j}$ describe the two-dimensional ferromagnetic Ising model at inverse temperature $K$. In particular, for $K>K_{c}$ there are two extremal Gibbs states with $E \sigma_{i, j}= \pm m \neq 0$.

\section{Variations on Model 1}

Model 1 is translation-invariant in the $x$ and $y$ directions, but has only finite extent in the $z$ direction, normal to the dividing surface $\partial \Lambda$. It may be of some interest to consider models with translations acting in all three directions. This may be obtained trivially by stacking up copies of Model 1 as layers which do not interact with each other. Somewhat less trivial is a model (Model 2) in which the $s$ spins of one layer are identified with the $u$ spins of the layer above. This model is on the lattice $\mathscr{L}_{2}$ consisting of sites $(i+1 / 2, j, k),(i, j+1 / 2, k)$ and $(i, j, k+1 / 2)$ with $i, j, k \in \mathbb{Z}$. We use $t$ 's to denote spins at the first two types of site and $s$ 's for the third type; spins take values \pm 1 . The formal Hamiltonian is

$$
\begin{aligned}
H= & J \sum_{i, j, k}\left(s_{i, j, k+1 / 2} t_{i, j+1 / 2, k} S_{i, j+1, k+1 / 2}+s_{i, j, k+1 / 2} t_{i+1 / 2, j, k} s_{i+1, j, k+1 / 2}\right. \\
& \left.+s_{i, j, k-1 / 2} t_{i, j+1 / 2, k} s_{i, j+1, k-1 / 2}+s_{i, j, k-1 / 2} t_{i+1 / 2, j, k} s_{i+1, j, k-1 / 2}\right) .
\end{aligned}
$$

We take $\Lambda=\mathscr{L}_{2} \cap\{z>0\}$, so that $\partial \Lambda=\mathscr{L}_{2} \cap\{z=0\}$.

In Model 2 there is no longer a local symmetry. However, the $t$ spins may still be "integrated out," leaving a model with formal Hamiltonian

$$
\begin{aligned}
H^{s}= & K \sum_{i, j, k}\left(s_{i, j, k+1 / 2} S_{i, j+1, k+1 / 2} S_{i, j, k-1 / 2} S_{i, j+1, k-1 / 2}\right. \\
& \left.+s_{i, j, k+1 / 2} S_{i+1, j, k+1 / 2} S_{i, j, k-1 / 2} S_{i+1, j, k-1 / 2}\right) .
\end{aligned}
$$

Using (3) as well as

$$
\sum_{t= \pm 1} t e^{J t\left(s_{1} s_{2}+s_{3} s_{4}\right)}=\text { const } \cdot s_{1} s_{2}+s_{3} s_{4} e^{K s_{1} s_{2} s_{3} s_{4}},
$$

we find that for any finite $A \subset \partial \Lambda, E\left(s_{i, j, 1 / 2} t_{A}\right)$ is a constant times a sum of expectations of products of $o d d$ numbers of $s$ spins (where $t_{A} \equiv \prod_{(x, y, z) \in A} t_{x, y, z}$ ). Now using the theory of Holsztyński and Slawny [3] on the system with the $t$ 's integrated out, we find that in the + phase at low temperature, the products of $s$ spins which have nonzero expectation are those which may be written as products of terms $s_{i, j, k+1 / 2} s_{i, j, k-1 / 2}$ (and in particular are even). Thus $E\left(s_{i, j, 1 / 2} t_{A}\right)=0$, which implies $E\left(s_{i, j, 1 / 2} \mid \mathscr{F}_{\partial A}\right)=0$, since linear combinations of the $t_{A}$ are dense in $L^{2}\left(\mathscr{F}_{\partial A}\right)$. On the other hand, $E\left(s_{i, j, 1 / 2} s_{i, j,-1 / 2}\right)>0$. Thus the + phase (which is an extremal Gibbs state) at low temperature fails to have the global Markov property.

While Model 2 is periodic in the $z$ direction, the action of translations is not transitive: the $s$ and $t$ spins are not equivalent, and even the $t$ spins at $(i+1 / 2, j, k)$ and $(i, j+1 / 2, k)$ are not related by a translation. We will now describe a third model (Model 3) in which translations act transitively on the lattice, but the spins are no longer just \pm 1 .

This time the lattice is the face-centred cubic lattice, which we write as $\mathscr{L}_{3}=\left\{(x, y, z) \in(1 / 2 \mathbb{Z})^{3}: x+y+z \in \mathbb{Z}\right\}$. At each site $\mathbf{x}$ there are three spin compo- 
nents $\sigma_{\mathbf{x}}^{1}, \sigma_{\mathbf{x}}^{2}, \sigma_{\mathbf{x}}^{3}$, each of which takes values \pm 1 . The formal Hamiltonian is $H=J \sum_{\mathbf{x} \in \mathscr{L}_{3}}\left\{\sigma_{\mathbf{x}}^{1}\left(\sigma_{\mathbf{x}+1 / 2(\mathbf{j}+\mathbf{k})}^{2}+\sigma_{\mathbf{x}+1 / 2(\mathbf{j}-\mathbf{k})}^{2}\right) \sigma_{\mathbf{x}+\mathbf{j}}^{1}+\sigma_{\mathbf{x}}^{1}\left(\sigma_{\mathbf{x}+1 / 2(\mathbf{i}+\mathbf{k})}^{3}+\sigma_{\mathbf{x}+1 / 2(\mathbf{i}-\mathbf{k})}^{3}\right) \sigma_{\mathbf{x}+\mathbf{i}}^{1}\right)$,

where $\mathbf{i}, \mathbf{j}, \mathbf{k}$ are $(1,0,0),(0,1,0)$ and $(0,0,1)$ respectively. Again $\Lambda=\mathscr{L}_{3} \cap\{z>0\}$, so $\partial \Lambda=\mathscr{L}_{3} \cap\{z=0\}$.

Model 3 is actually composed of four separate copies of Model 2, not interacting with each other. One of these copies consists of the $\sigma^{1}$ components on the sublattice $\mathbb{Z}^{3}$ (acting as $s$ spins), the $\sigma^{2}$ components on the sublattice $\mathbb{Z}^{3}+(0,1 / 2,1 / 2)$, the $\sigma^{3}$ components on the sublattice $\mathbb{Z}^{3}+(1 / 2,0,1 / 2)$, and nothing on the fourth sublattice $\mathbb{Z}^{3}+(1 / 2,1 / 2,0)$. The other copies are translates of this one by $(0,1 / 2,1 / 2),(1 / 2,0,1 / 2)$ and $(1 / 2,1 / 2,0)$. Thus the results for Model 2 carry over to Model 3.

\section{Open Questions}

We conclude with some questions that remain open.

(1) Does the global Markov property hold whenever the Gibbs state for $\Phi$ is unique?

The only known result in this direction is that strong uniqueness [1] implies the global Markov property. On the other hand, in our examples the nonuniqueness of the Gibbs state is a key ingredient in the failure of the global Markov property.

(2) If $\mathscr{L}$ is two-dimensional, does the Markov property (1) hold when $\Lambda$ is a half-plane?

If we take the cross-section of the lattice of Model 1 for $y=0$ and make $J$ infinite (i.e. replace the interaction by the constraints $s_{i, 0} t_{i+1 / 2,0} s_{i+1,0}$ $=u_{i, 0} t_{i+1 / 2,0} u_{i+1,0}=1$ ), we obtain an example which is essentially equivalent to von Weizsäcker's [4]. This example would not violate the global Markov property if $J$ were finite, because a one-dimensional system with bounded finite-range interactions can have no long-range order. Adding to the lattice in the $z$ direction would not appear to help; it seems the long-range order must "propagate along $\partial \Lambda$, , which requires $\partial \Lambda$ to be at least two-dimensional.

(3) Does the global Markov property hold for extremal Gibbs states with spins of \pm 1 and an interaction invariant under a transitive action of translations?

There is a good reason why to obtain an example (Model 3) with translations acting transitively on the lattice, we had to use spins with more than one component. For spins taking values \pm 1 with a ferromagnetic interaction invariant under a transitive action of translations, the Holsztynski-Slawny theory [3] applies: $E\left(\sigma_{A}\right)>0$ in the + phase at low temperature if and only if $\sigma_{A}$ is a product of translates of $\sigma_{A}$, where $\Delta$ is the "greatest common divisor" of the bonds. Suppose $A \Subset \dot{\Lambda}$ and $E\left(\sigma_{A} \mid \mathscr{F}_{\mathscr{L} \backslash \Lambda}\right) \neq 0$. Then $E\left(\sigma_{A} \sigma_{B}\right) \neq 0$ for some $B \subset \mathscr{L} \backslash \Lambda$, and $\sigma_{A} \sigma_{B}$ is a product of translates of $\sigma_{\Delta}$. If we restrict the product to translates contained in $\Lambda \cup \partial \Lambda$, we obtain $E\left(\sigma_{A} \sigma_{C}\right) \neq 0$ with $C \subset \partial \Lambda$, i.e. $E\left(\sigma_{A} \mid \mathscr{F}_{\partial \Lambda}\right) \neq 0$ (all these expectations being in the + phase at low temperature). This does not prove the global Markov property, but shows that it will not be violated in quite the same way as in our examples. In 
particular, it would probably be much harder to prove that $E\left(\sigma_{A} \mid \mathscr{F}_{\mathscr{L} \backslash A}\right)$ $\neq E\left(\sigma_{A} \mid \mathscr{F}_{\partial A}\right)$ without the latter being zero.

Acknowledgements. I would like to thank Herbert Spohn for providing me with ref. [4], Sheldon Goldstein for useful discussions, and Joel Lebowitz for his hospitality at Rutgers University.

\section{References}

1. Follmer, H.: On the global Markov property. In:Quantum fields - algebras, processes. L. Streit (ed.). Berlin, Heidelberg, New York: Springer 1980

2. Goldstein, S.: Remarks on the global Markov property. Commun. Math. Phys. 74, 223-234 (1980)

3. Holsztyński, W., Slawny, J.: Phase transitions in ferromagnetic spin systems at low temperatures. Commun. Math. Phys. 66, 147-166 (1979)

4. von Weizsäcker, H.: A simple example concerning the global Markov property of lattice random fields. In: Eighth Winter School on Abstract Analysis (1980)

Communicated by J. Fröhlich

Received January 16, 1986

Note added in proof. For an example with unbounded interaction see C. Kessler. Publ. RIMS Kyoto 21, 877-888 (1985). 
